\title{
Erratum to: Effectiveness of risedronate and alendronate on nonvertebral fractures: an observational study through 2 years of therapy
}

\author{
R. Lindsay $\cdot$ N. B. Watts $\cdot$ J. L. Lange $\cdot$ P. D. Delmas • \\ S. L. Silverman
}

Published online: 29 May 2013

(C) International Osteoporosis Foundation and National Osteoporosis Foundation 2013

\section{Erratum to: Osteoporos Int}

DOI 10.1007/s00198-013-2332-7

The legends for Figs. 2 and 3 appeared in the correct places but were accompanied by the wrong illustrations: Fig. 2 legend by Fig. 3 illustrations, and Fig. 3 legend by Fig. 2 illustrations. The two figures are reproduced here in their correct form.

The online version of the original article can be found at http://dx.doi.org/ 10.1007/s00198-013-2332-7.

R. Lindsay $(\bowtie)$

Helen Hayes Hospital, 51-55 Route 9W,

West Haverstraw, NY 10993, USA

e-mail: lindsayr@helenhayeshosp.org

N. B. Watts

Mercy Health Osteoporosis and Bone Health Services, Cincinnati,

$\mathrm{OH}$, USA

J. L. Lange

Procter \& Gamble, Mason, OH, USA

P. D. Delmas

INSERM Unit 403, Université Claude Bernard Lyon 1, Lyon,

France

S. L. Silverman

Cedars-Sinai Medical Center and University of California, Los

Angeles, CA, USA 
Fig. 2 Hip fracture rate. $95 \%$ confidence intervals around point estimate. Note the early separation of the two cohorts with a lower fracture rate for risedronate than for alendronate during the early phase (6-12 months) of treatment

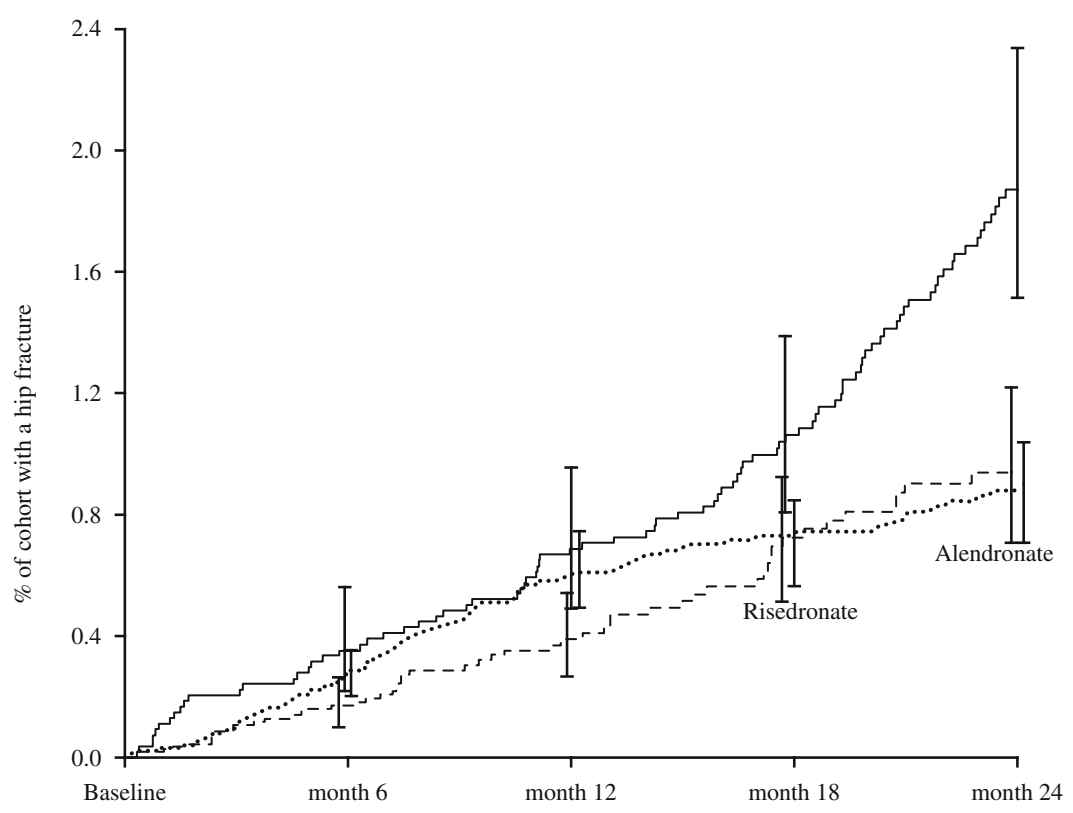

Patients at risk (fracture events) by cohort at 6 month intervals

\begin{tabular}{lccccc}
\hline Referent & $5,390(0)$ & $5,371(19)$ & $5,353(37)$ & $4,428(55)$ & $3,621(88)$ \\
Risedronate & $12,215(0)$ & $8,420(19)$ & $5,275(34)$ & $3,572(48)$ & $2,680(56)$ \\
Alendronate & $21,615(0)$ & $15,239(55)$ & $9,745(95)$ & $6,877(105)$ & $5,144(115)$ \\
\hline
\end{tabular}

Fig. 3 Nonvertebral fracture rate. $95 \%$ confidence intervals around point estimate. Note the early separation of the two cohorts with a lower fracture rate for risedronate than for alendronate during the early phase (6-12 months) of treatment

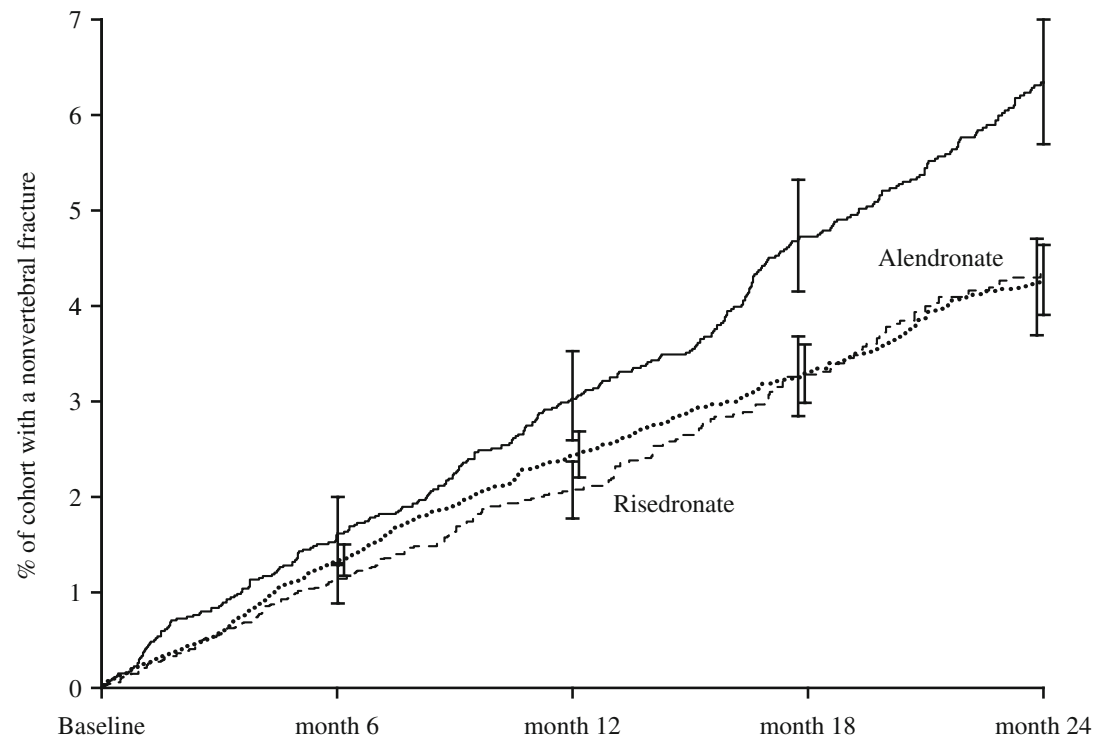

Patients at risk (fracture events) by cohort at 6 month intervals

\begin{tabular}{lccccc}
\hline Referent & $5,390(0)$ & $5,307(87)$ & $5,227(163)$ & $4,275(245)$ & $3,466(311)$ \\
Risedronate & $12,215(0)$ & $8,346(128)$ & $5,205(190)$ & $3,505(243)$ & $2,619(278)$ \\
Alendronate & $21,615(0)$ & $15,086(266)$ & $9,585(401)$ & $6,731(471)$ & $5,002(531)$ \\
\hline
\end{tabular}

